\title{
Poor Prognostic Factors for Surgical Treatment of Spinal Intramedullary Ependymoma (World Health Organization Grade II)
}

\author{
Osahiko Tsuji ${ }^{1}$, Narihito Nagoshi ${ }^{1}$, Ryota Ishii ${ }^{2}$, Satoshi Nori ${ }^{1}$, Satoshi Suzuki ${ }^{1}$, Eijiro Okada ${ }^{1}$, \\ Nobuyuki Fujita ${ }^{3}$, Mitsuru Yagi ${ }^{1}$, Morio Matsumoto ${ }^{1}$, Masaya Nakamura ${ }^{1}$, Kota Watanabe ${ }^{1}$ \\ ${ }^{I}$ Department of Orthopaedic Surgery, Keio University School of Medicine, Tokyo, Japan \\ ${ }^{2}$ Biostatistics Unit, Clinical and Translational Research Center, Keio University Hospital, Tokyo, Japan \\ ${ }^{3}$ Department of Orthopaedic Surgery, Fujita Health University, Toyoake, Japan
}

Study Design: Single-center retrospective study.

Purpose: We aimed to explore the postoperative prognostic factors for spinal intramedullary ependymoma.

Overview of Literature: Ependymoma (World Health Organization grade II) is the most frequent intramedullary spinal tumor and is treated by total resection. However, postoperative deterioration of motor function occasionally occurs.

Methods: Eighty patients who underwent surgical resection at Keio University and Fujita Health University in Tokyo, Japan between 2003 and 2015 with more than 2 years of follow-up were enrolled. A good surgical result was defined as an improvement in the modified McCormick Scale score by one grade or more or having the same clinical grade as was observed preoperatively. Meanwhile, a poor result was defined as a reduction in the McCormick Scale score of one grade or more or remaining in grade IV or $\mathrm{V}$ at final follow-up. Univariate and multivariate logistic regression analyses of the following factors were performed in the two groups: sex, age, preoperative Visual Analog Scale (VAS), tumor location, the extent of tumor resection, hemosiderin caps, cavity length, and tumor length on magnetic resonance imaging.

Results: At final follow-up, 15 patients were included in the poor results group and 65 in the good results group. In the univariate analysis, the factors related to poor results were as follows: higher age, preoperative McCormick Scale score severity, higher preoperative VAS, thoracic location, hemosiderin capped, and non-gross total resection (GTR). A multiple logistic regression analysis was conducted and showed that age, worse preoperative McCormick Scale score, and non-GTR were significant factors for poor prognosis.

Conclusions: The independent risk factors for motor deterioration after ependymoma resection were age, worse preoperative McCormick Scale score, and non-GTR. Early surgery for patients with even mild neurological disorders could facilitate functional outcomes. These results may contribute to determining the optimal timing of surgery for spinal intramedullary ependymoma.

Keywords: Spinal intramedullary ependymoma; Surgical outcome; Prognostic factor; McCormick Scale

This is the 2019 APSS-Asian Spine Journal Best Paper Award.

Received Feb 11, 2020; Revised Feb 26, 2020; Accepted Mar 1, 2020

Corresponding author: Kota Watanabe

Department of Orthopaedic Surgery, 35 Shinanomachi, Shinjuku-ku, Keio University School of Medicine, Tokyo, 160-8582, Japan

Tel: +81-3-5363-3812, Fax: +81-3-3353-6597, E-mail: kw197251@keio.jp 


\section{Introduction}

Spinal cord intramedullary tumor is an extremely rare disease with an incidence of 1.1 per 100,000 people, accounting for $2 \%-4 \%$ of central nervous system tumors [1]. Most spinal cord intramedullary tumors are gliomas (ependymoma or astrocytoma); the incidence of ependymoma is high in adults, whereas astrocytoma is more common in children [2,3]. Spinal intramedullary ependymoma is a prevalent type of rare spinal cord tumor and exhibits various clinical features depending on location and malignancy $[4,5]$. The 2007 World Health Organization (WHO) classification of tumors of the central nervous system classified ependymal tumors into four types: subependymoma (grade I), myxopapillary ependymoma (grade I), ependymoma (grade II, cellular, papillary, clear cell, and tanycytic), and anaplastic ependymoma (grade III) [6]. The 2016 WHO classification update added a new type of ependymoma defined by genetic abnormalities (RELA fusion-positive type) [7]. The 2007 WHO grade II spinal intramedullary ependymoma generally shows a slow growth pattern, and the prognosis is good after gross total excision; however, recurrence can be extremely delayed even after total resection [1,8-10]. In 2008, we reported the surgical outcomes of 33 patients with intramedullary grade II ependymomas (surgically treated in our institute between 1994 and 2003) and found that four patients with thoracic location and/or partial tumor resection had worsened postoperative ambulation evaluated by Frankel grade [11]. The sample size of our previous study was small, and the factors related to poor ambulation were derived from qualitative data. In this study, we used a larger sample size to retrospectively analyze the poor prognostic factors for surgically treated WHO grade II spinal intramedullary ependymomas in a single-center.

\section{Materials and Methods}

\section{Participants}

We retrospectively reviewed the data of 1,020 consecutive patients who underwent spinal cord tumor resection surgery at our institution between January 2003 and December 2015. Clinical data of each patient were collected from patients' charts and operative records according to our institution's ethical guidelines. This study was approved by the ethics committee of Keio University School of Medicine (approval no., 20110222). Informed consent was obtained in the form of opt-out on the website. Those who rejected were excluded. Among them, 333 patients presented with intramedullary tumors. A total of $80 \mathrm{pa}-$ tients (54 men and 26 women) with intramedullary spinal ependymoma (WHO grade II) underwent tumor resection surgery with at least 2 years of outpatient follow-up (mean follow-up, 7.4 years; range, 2-11 years). The patients' mean age was $50.5 \pm 15.0$ years (range, $21-82$ years). Spinal intramedullary ependymoma was diagnosed by magnetic resonance imaging (MRI) in all cases.

\section{Surgery}

Three surgeons performed the surgery during the survey period. All patients underwent surgery under an operative microscope via the posterior approach with intraoperative neurophysiological monitoring of motor-evoked potentials (MEPs). Our treatment policy for spinal intramedullary ependymomas was to attempt total resection as much as possible. However, when MEPs were attenuated, we temporarily suspended the surgery; if the potentials decreased in almost all of the monitored muscles, we evacuated the whole tumor, even at the partial resection state. Furthermore, if the border between the normal cord and tumor surface was unclear, total resection was discontinued and we removed as much of the tumor as possible or performed biopsy alone. Patients with cervical tumors were operated while in the prone Concorde position fixed to the operating table by a three-pin head holder Mayfield (Integra Life Sciences, Plainsboro, NJ, USA) system; those with upper thoracic (T1-4) tumors were placed in the prone position using both Mayfield (Integra Life Sciences) and a Relton-Hall frame; and those with thoracic and thoracolumbar tumors were placed in a prone position using a Relton-Hall frame. We asked the anesthetists to maintain arterial blood pressure at $80-100 \mathrm{~mm} \mathrm{Hg}$ throughout the surgery. Following laminoplasty in the case of cervical tumors or laminectomy in both thoracic and thoracolumbar tumors, tumor location was confirmed by intraoperative ultrasonography and then the tumor was approached from the midline of the cord (myelotomy via the posterior median sulcus). We employed microsurgical procedures based on previous works [11,12]. After sufficient cephalocaudal range of myelotomy, dissection of the tumor surface was carried out from the dorsal to the ventral side. When the tumor was large, the tumor within the 
capsule was debulked using a Cavitron Ultrasonic Surgical Aspirator (Integra Life Sciences Corp., Plainsboro, NJ, USA), and then resection was performed after detaching the ventral end of the tumor from the normal cord tissue. After tumor resection, the cord was reintegrated to the pia mater at the posterior median sulcus using a 9-0 nylon suture with several knots. The extent of surgical resection was defined as follows based on previous studies $[11,13]$ : gross total resection (GTR) as no visual evidence of tumor on the surgical field of the operating microscope, near total resection (NTR) as $<10 \%$ residual tumor mass after surgery, and subtotal resection (STR) as removal of $50 \%-90 \%$ of tumor tissue by the end of surgery.

\section{Data collection}

We evaluated the ambulatory ability of participants before and 2 years after tumor resection using the modified McCormick Scale (grades I-V: I, normal gait; II, mild gait disturbance not requiring support; III, gait with support; IV, assistance required; and V, wheelchair needed) [14,15]. The good results group was defined as patients with a final follow-up McCormick Scale score of $\geq 1$ grade, having the same clinical grade as that prior to the operation, or staying in grades I-III (G-group). The poor results group were defined as patients with motor paralysis and worsening by $\geq 1$ on the McCormick Scale or staying in grade IV or V until final follow-up (P-group). The surveyed items were as follows: age, gender, pre- and postoperative modified McCormick Scale score, preoperative Visual Analog Scale (VAS), tumor location (including thoracic level), extent of tumor resection (GTR or NTR/STR), hemosiderin caps, length of the syrinx, and tumor length on MRI. A univariate analysis was initially performed; using factors with a $p$-value of $<0.2$, a multivariate logistic regression analysis was performed on the two groups.

\section{Statistical analysis}

Statistical analysis was performed using IBM SPSS Statistics ver. 25.0 (IBM Corp., Armonk, NY, USA). Continuous variables were expressed as means \pm standard deviation (SD), while categorical variables were expressed as percentages. A univariate analysis (Mann-Whitney $U$-test for continuous variables and chi-square test for categorical variables) was initially performed. Then, using factors with a $p$-value of $<0.2$ in the univariate analysis, a multi- variate logistic regression analysis was performed on the two groups to examine the association between functional deterioration and individual risk factors and estimated the odds ratio (OR) and 95\% confidence interval (95\% CI). A $p$-value of $<0.05$ was considered significant. Data were presented as mean $\pm \mathrm{SD}$ unless otherwise indicated.

\section{Results}

\section{Preoperative demographic data}

Table 1 summarizes the baseline demographics of the 80 patients who underwent tumor resection surgery for spinal intramedullary ependymoma. Of them, 55 (68.8\%) were men, and 35 were women, with a mean age of $50.5 \pm 15.0$ years (range, $21-82$ years). The mean VAS was $30.4 \pm 27.5$ (range, $0-100$ ), and the mean follow-up period was $5.8 \pm 2.2$ years (range, $2-11$ years). All patients underwent surgery via a posterior approach, and 70 underwent GTR of the tumor (87.5\%). A spinal intramedullary ependymoma was detected in 44 patients (55.0\%) at the cervical level and $36(45.0 \%)$ at the thoracic level. MRI findings from imaging of the sagittal section revealed that the aver-

Table 1. Baseline characteristics of all participants

\begin{tabular}{lc}
\hline Characteristic & \multicolumn{1}{l}{ Value } \\
\hline Preoperative Visual Analog Scale of pain & $30.4 \pm 27.5(0-100)$ \\
\hline Surgical approach (posterior) & $100(80)$ \\
\hline Extent of resection & \\
\hline Gross total resection & $87.5(70)$ \\
\hline Near total resection/sub-total resection & $12.5(10)$ \\
\hline Location of tumor & \\
\hline Cervical & $55.0(44)$ \\
\hline Thoracic & $45.0(36)$ \\
\hline Tumor length (vertebra length) & $2.8 \pm 1.6(0.5-8)$ \\
\hline Length of syrinx (vertebra length) & $6.3 \pm 4.2(0-17)$ \\
\hline Hemosiderin cap (positive) & $65.0(52)$ \\
\hline Baseline modified McCormick Scale & \\
\hline I (normal gait) & $45.0(36)$ \\
\hline II (mild gait disturbance) & $25.0(20)$ \\
\hline III (gait with support) & $11.2(9)$ \\
\hline IV (assistance required) & $12.5(10)$ \\
\hline V (wheel chair needed) & $6.3(5)$ \\
\hline Values are presented as mean \pm standard deviation (range), \%, or \% (number of \\
\hline cases).
\end{tabular}




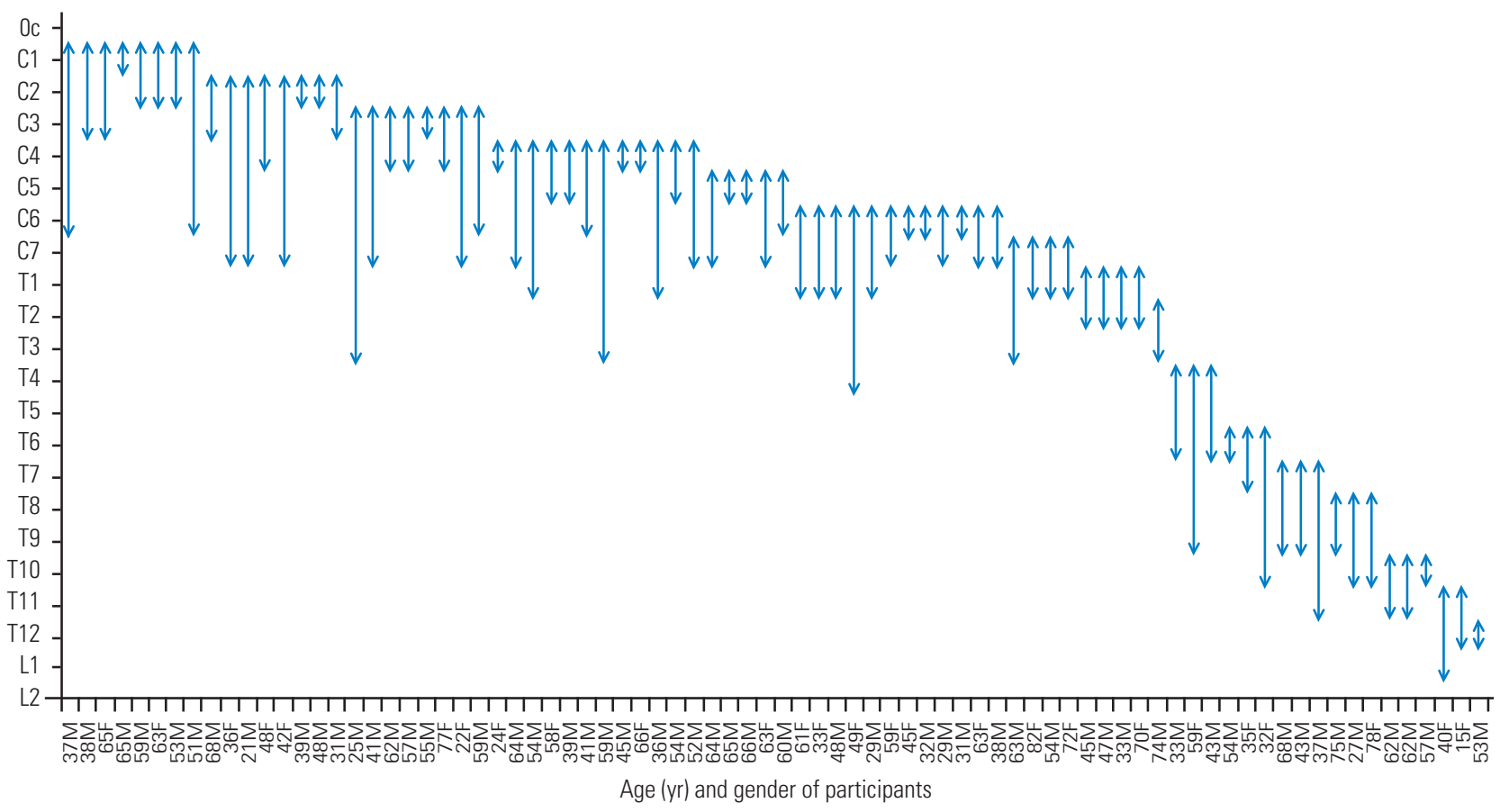

Fig. 1. Distribution of spinal intramedullary ependymoma. Arrowheads indicate the rostral and caudal ends of tumors. The numbers and female/male on the horizontal axis represent age and gender (female or male) of each participant, respectively. The mean tumor length of all participants was $2.8 \pm 1.7$ vertebrae in length. $\mathrm{M}$, male; $\mathrm{F}$, female.

age tumor length was $2.8 \pm 1.6$ vertebrae in length (range, $0.5-8$ ) (Fig. 1), while the average syrinx size was $6.3 \pm 4.2$ vertebrae in length (range, $0-17$ ). A hemosiderin cap sign was noted on the rostral and/or caudal edge of the tumor in 52 patients $(65.0 \%)$ on MRI. The baseline modified McCormick Scale score was grade I in 36 patients (45.0\%), grade II in 20 patients $(25.0 \%)$, grade III in nine patients (11.2\%), grade IV in 10 patients $(12.5 \%)$, and grade $\mathrm{V}$ in five patients $(6.3 \%)$.

\section{Tumor distribution}

The spinal location and distribution of the tumor for each patient are shown in Fig. 1. The mean tumor length of all patients was 2.8 vertebrae in length. Spinal intramedullary ependymomas can extend upward to 3-4 vertebrae and are usually confined to five vertebral segments $[16,17]$. Upon defining the giant ependymoma as more than five vertebrae in length, 15 patients in our series were found to have a giant ependymoma (Fig. 1), of which six were five vertebrae in length, seven were six vertebrae in length, one was seven vertebrae in length, and another one was eight vertebrae in length (Fig. 1).

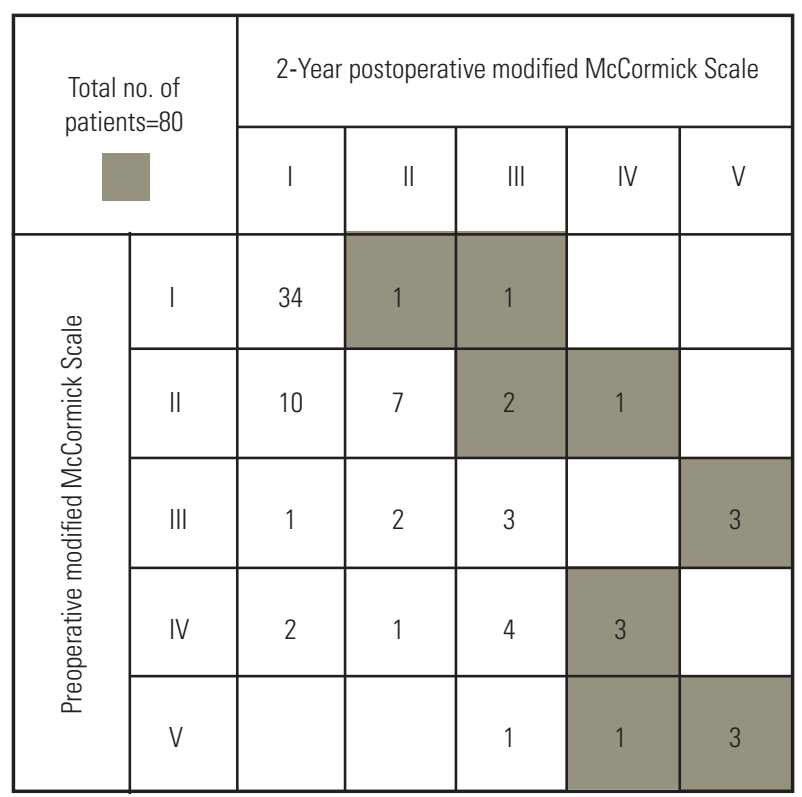

Fig. 2. Pre- and postoperative walking ability evaluated by modified McCormick Scale. We evaluated the pre- and postoperative ambulatory abilities and stratified 65 patients as G-group and 15 as P-group. P-group is indicated by the gray-colored panel. 
Table 2. Univariate analysis comparing G- and P-group

\begin{tabular}{|c|c|c|c|}
\hline Variable & G-group ( $n=65)$ & P-group $(n=15)$ & $p$-value \\
\hline Age (yr) & $48.4 \pm 13.9$ & $56.8 \pm 18.0$ & $0.083^{\mathrm{al}}$ \\
\hline Gender ( $\%$ male) & 67.7 & 66.7 & $0.582^{b)}$ \\
\hline Preoperative modified McCormick Scale (no. of cases) & & & $<0.001^{\text {b) }}$ \\
\hline । & 34 & 2 & \\
\hline ॥ & 17 & 3 & \\
\hline III & 6 & 3 & \\
\hline IV & 7 & 3 & \\
\hline V & 1 & 4 & \\
\hline Postoperative modified McCormick Scale (no. of cases) & & & $<0.001^{\text {b) }}$ \\
\hline । & 47 & 0 & \\
\hline$\|$ & 10 & 1 & \\
\hline III & 8 & 3 & \\
\hline IV & 0 & 4 & \\
\hline V & 0 & 6 & \\
\hline Preoperative Visual Analog Scale (mm) & $28.4 \pm 26.1$ & $39.2 \pm 32.3$ & $0.265^{\text {a) }}$ \\
\hline Thoracic location of tumor (\%) & 41.5 & 60.0 & $0.195^{b /}$ \\
\hline Extent of tumor resection (\% gross total resection) & 93.8 & 60.0 & $<0.001^{\text {a) }}$ \\
\hline Hemosiderin cap positive on MRI (\%) & 60.0 & 86.7 & $0.051^{b /}$ \\
\hline Length of syrinx on MRI (no. of vertebra) & $6.1 \pm 4.2$ & $7.4 \pm 4.2$ & $0.268^{a)}$ \\
\hline Tumor length on MRI (no. of vertebra) & $2.8 \pm 1.7$ & $2.9 \pm 1.2$ & $0.401^{\text {a) }}$ \\
\hline
\end{tabular}

Values are presented as mean \pm standard deviation, \%, or number of cases, unless otherwise stated. G-group: the cases with "good" surgical results at final followup defined as McCormick Scale improved 1 grade or more, remaining the same grade as preoperative status. P-group: those with "poor" results defined as with motor paralysis as one or more McCormick Scale deterioration, or stayed in IV or V at final follow-up.

$\mathrm{MRI}$, magnetic resonance imaging.

${ }^{a} B y$ Mann-Whitney $U$-test for continuous variables. ${ }^{b} B y$ chi-square test for categorical variables.

\section{Pre- and postoperative walking ability and univariate analysis: predictors for poor outcome}

Of the 80 patients, $65(81.3 \%)$ were categorized as Ggroup and $15(18.7 \%)$ as P-group at 2-year follow-up (Fig. 2). For each group, a univariate analysis (Mann-Whitney $U$-test for continuous variables and chi-square test for categorical variables) was performed (Table 2). According to our criteria ( $p<0.2$ in univariate analysis), age $(p=0.083)$, preoperative McCormick Scale score (higher: worse, $p<0.001)$, thoracic location ( $p=0.195)$, extent of tumor resection (non-GTR, $p<0.001$ ), and positive hemosiderin cap $(p=0.051)$ were significant predictors of poor outcome (P-group) (Table 2). Other variables (preoperative VAS, tumor length, and syrinx length) did not satisfy our criteria.
Table 3. Multivariate logistic regression analysis

\begin{tabular}{lcc} 
Variable & $\begin{array}{c}\text { Odds ratio } \\
\text { (95\% confidence } \\
\text { interval) }\end{array}$ & $p$-value \\
\hline Age (older) & $1.054(1.001-1.11)$ & 0.046 \\
\hline Preoperative modified McCormick Scale & & 0.007 \\
\hline 1 & Reference & \\
\hline 2 & $2.48(0.33-18.36)$ & \\
\hline 3 & $3.82(0.42-34.65)$ & \\
\hline 4 & $6.67(0.80-55.6)$ & \\
\hline 5 & $36.74(2.12-636.38)$ & \\
\hline Extent of resection & & \\
\hline Near total resection/sub-total resection & Reference & \\
\hline Gross total resection & $0.083(0.014-0.51)$ & 0.007 \\
\hline
\end{tabular}




\section{Poor prognostic factors evaluated by multivariate lo- gistic analysis}

All variables that yielded a $p$-value of $<0.2$ in the univariate analyses were evaluated using multiple logistic regression analysis and a forward stepwise procedure. According to the final model, higher age (OR, 1.054; 95\% CI, $1.001-1.11 ; p=0.046)$ and worse preoperative McCormick Scale score (OR, 2.10; $p=0.007)$ were poor independent prognostic factors of spinal intramedullary ependymoma in the P-group (Table 3). GTR was a good independent prognostic factor for spinal intramedullary ependymoma (reference, NTR/STR; OR, 0.083; 95\% CI, 0.014-0.51; $p=0.007$ ) (Table 3 ). With regard to higher age, the calculated OR for an additional 10 years of age was 1.69.

\section{Discussion}

In the present study, we evaluated the predictors for postoperative poor ambulation following surgical resection of spinal intramedullary ependymoma. The results of multiple logistic regression analysis revealed that age, worse preoperative McCormick Scale score, and non-GTR (NTR or STR) were poor independent prognostic factors. As the results from the multivariate analysis could halt the intervention of confounders, these factors are deemed to accurately reflect prognostic predictors that affect the postsurgical outcome of spinal intramedullary ependymoma.

Recently, several studies have reported the prognostic factors for surgical resection of spinal ependymoma. Klekamp [12] analyzed a total of 100 patients with spinal intramedullary ependymoma who were surgically treated at two hospitals in Germany between 1980 and 2014. They reported that a low preoperative McCormick Scale score was the strongest predictor for achieving GTR, and that thoracic location, age, longer clinical history, and presence of tumor hemorrhage were risk factors for permanent neurological morbidity [12]. Wostrack et al. [18] performed a multicenter retrospective study of 158 patients with spinal ependymoma (44 patients with grade I, 105 with grade II, and nine with grade III). They evaluated the prognostic factors by multivariate analysis and consequently found that GTR, grade II tumor, and lower $\mathrm{Ki}-67$ index were independent prognostic factors for 5 -year progression-free survival [18]. The GTR and grade II results were similar to those of our study, and a higher Ki-67 index was highlighted because their study included nine patients with grade III ependymoma. Kumar and Banerjee [13] carried out a prospective clinical study to evaluate the functional outcome of 43 patients with spinal cord intramedullary tumor, including 21 with intramedullary spinal ependymoma, and concluded that a low preoperative McCormick Scale score and GTR were key factors for good functional outcome and survival. Taken together, the poor prognostic factors (higher age, lower preoperative McCormick Scale score, and non-GTR) found in the current study are compatible with those of previous studies. The current study used the largest sample size $(n=80)$ for a single-center retrospective study, eliminating the potential disparities between different centers. Multicenter studies are superior to single institutional research studies because of the lack of potential data bias in the characteristics of a single institution and the minimization of researcher subjectivity. By contrast, one of the drawbacks of multicenter studies is that data quality tends to deteriorate due to the differences in data accuracy and interpretation between each participating facility. Our study, as a single-center setting with the regularization of the operators' skill, eliminated this potential disadvantage of a multicenter study. However, our study was still limited by a relatively small sample size because of the rarity of this type of tumor. Hence, further prospective studies with a larger sample size is warranted to establish solid treatment guidelines for spinal intramedullary ependymoma.

This study had several limitations that should be discussed. First, this was a single-center retrospective study with potential selection bias, inevitably confining the evidence level. Second, surgical procedures were performed by more than one surgeon (total three surgeons in our department) during the survey period (13 years), which could affect the variation of surgical outcomes due to individual surgeons' proficiency. Third, non-GTR as a prognostic factor in the current study was the result of the deterioration of intraoperative MEPs. Whether GTR is successfully achieved or not is dependent upon the difficulty of tumor dissection and the adhesive change between the tumor and normal cord; however, it is impracticable to preoperatively predict the degree of adhesive change of tumor surfaces. To overcome this difficulty, intraoperative 5-aminolevulinic acid (5-ALA)-assisted resection of spinal cord ependymomas has recently been recommended [19]. We may utilize this 5-ALA-guided surgery at our institute in the near future. Finally, the statistical power of this study was not strong because of the 
small sample size. Indeed, the positive hemosiderin cap findings on MRI were almost statistically significant at the univariate analysis but were conclusively excluded by a stepwise procedure in the multivariate analysis. However, as stated above, the number of patients with surgically treated spinal intramedullary ependymoma analyzed in previous multicenter studies was, at most, around 100, and the prognostic factors in those studies were compatible those reported in our study $[12,18]$. Although further investigation with larger sample size is necessary to clarify more precise factors for a good prognosis of spinal intramedullary ependymoma, it was deemed significant to report the preoperative poor prognostic factors for these tumors.

\section{Conclusions}

In conclusion, the independent risk factors for motor deterioration after ependymoma resection were age, worse preoperative McCormick Scale score, and non-GTR. Early diagnosis and surgery for patients with even mild neurological disorders could facilitate functional outcomes. Our data may contribute to determining the optimal timing of surgery for spinal intramedullary ependymoma.

\section{Conflict of Interest}

No potential conflict of interest relevant to this article was reported.

\section{Author Contributions}

OT designed the study, and wrote the initial draft of the manuscript. $\mathrm{NN}, \mathrm{MM}, \mathrm{MN}$, and $\mathrm{KW}$ was responsible for designing the study protocol, recruiting participants. RI played an important role in the statistical verification, and contributed to the preparation of the manuscript. SN, SS, EO, NF, and MY contributed to analysis and interpretation of data, and assisted in the preparation of the manuscript. KW was responsible for all working related to this submission as corresponding author. Also, all authors approved the final version manuscript and agreed to be accountable for all aspects of the work.

\section{References}

1. Karikari IO, Nimjee SM, Hodges TR, et al. Impact of tumor histology on resectability and neurological outcome in primary intramedullary spinal cord tumors: a single-center experience with 102 patients. Neurosurgery 2011;68:188-97.

2. Merchant TE, Pollack IF, Loeffler JS. Brain tumors across the age spectrum: biology, therapy, and late effects. Semin Radiat Oncol 2010;20:58-66.

3. Nishikawa R. Pediatric and adult gliomas: how different are they? Neuro Oncol 2010;12:1203-4.

4. Stein BM, McCormick PC. Intramedullary neoplasms and vascular malformations. Clin Neurosurg 1992;39:361-87.

5. Cristante L, Herrmann HD. Surgical management of intramedullary spinal cord tumors: functional outcome and sources of morbidity. Neurosurgery 1994;35:69-74.

6. Louis DN, Ohgaki H, Wiestler OD, et al. The 2007 WHO classification of tumours of the central nervous system. Acta Neuropathol 2007;114:97-109.

7. Louis DN, Perry A, Reifenberger G, et al. The 2016 World Health Organization Classification of Tumors of the Central Nervous System: a summary. Acta Neuropathol 2016;131:803-20.

8. Brotchi J. Intrinsic spinal cord tumor resection. Neurosurgery 2002;50:1059-63.

9. Reni M, Gatta G, Mazza E, Vecht C. Ependymoma. Crit Rev Oncol Hematol 2007;63:81-9.

10. Volpp PB, Han K, Kagan AR, Tome M. Outcomes in treatment for intradural spinal cord ependymomas. Int J Radiat Oncol Biol Phys 2007;69:1199-204.

11. Nakamura M, Ishii K, Watanabe K, et al. Surgical treatment of intramedullary spinal cord tumors: prognosis and complications. Spinal Cord 2008;46:282-6.

12. Klekamp J. Spinal ependymomas. Part 1: Intramedullary ependymomas. Neurosurg Focus 2015;39:E6.

13. Kumar R, Banerjee S. Management and functional outcome of intramedullary spinal cord tumors: a prospective clinical study. Asian J Neurosurg 2014;9:17781.

14. McCormick PC, Torres R, Post KD, Stein BM. Intramedullary ependymoma of the spinal cord. J Neurosurg 1990;72:523-32.

15. Matsuyama Y, Sakai Y, Katayama Y, et al. Surgical results of intramedullary spinal cord tumor with spinal cord monitoring to guide extent of resection. J Neurosurg Spine 2009;10:404-13. 
16. Zimmerman RA, Bilaniuk LT. Imaging of tumors of the spinal canal and cord. Radiol Clin North Am 1988;26:965-1007.

17. Kahan H, Sklar EM, Post MJ, Bruce JH. MR characteristics of histopathologic subtypes of spinal ependymoma. AJNR Am J Neuroradiol 1996;17:143-50.

18. Wostrack M, Ringel F, Eicker SO, et al. Spinal ep- endymoma in adults: a multicenter investigation of surgical outcome and progression-free survival. J Neurosurg Spine 2018;28:654-62.

19. Moreno RG, Garcia LMB, Bastidas HI, et al. Fluorescence guided surgery with 5-aminolevulinic acid for resection of spinal cord ependymomas. Asian Spine J 2019;13:119-25. 